\title{
ON THE EXISTENCE OF PERIODIC AND EVENTUALLY PERIODIC SOLUTIONS OF A FLUID DYNAMIC FORCED HARMONIC OSCILLATOR
}

\author{
CHARLIE H. COOKE \\ Department of Mathematical Sciences \\ 01d Dominion University \\ Norfolk, Virginia 23508 \\ (Received November 18,1985$)$
}

ABSTRACT. For certain flow regimes, the nonlinear differential equation $\ddot{Y}=F(Y)-G, \quad Y \geq 0, G>0$ and constant,

models qualitatively the behaviour of a forced, fluid dynamic, harmonic oscillator which has been a popular department store attraction. The device consists of a ball oscillating suspended in the vertical jet from a household fan. From the postulated form of the mode1, we determine sets of attraction and exploit symmetry properties of the system to show that all solutions are either initially periodic, with the ball never striking the fan, or else eventually approach a periodic limit cycle, after a sufficient number of bounces away from the fan.

KEY WORDS AND PHRASES. Nonlinear fluid dynamics, forced harmonic oscillator, eventually periodic motion, sets of attraction.

1980 AMS SUBJECT CLASSIFICATION CODES. $34 \mathrm{C} 15$ and $34 \mathrm{C} 25$.

1. INTRODUCTION.

Our intent is to study the behaviour of solutions to the non-linear, second order equation

$$
\ddot{Y}=F(Y)-G, \quad Y \geqq 0, \quad G \text { constant, }
$$

with the function $F(Y)$ positive, continuous, monotone non-increasing, and bounded; where

$$
\lim _{Y \rightarrow \infty} F(Y)=0
$$

We assume further that the equation

$$
F(Y)-G=0
$$

has a single root located at $Y=Y_{S}$, and that $F(Y)$ assumes its maximum for the last time, as $\mathrm{Y}$ increases, at $\mathrm{Y}=\mathrm{Y}_{\mathrm{M}}$, where $\mathrm{Y}_{\mathrm{S}}>\mathrm{Y}_{\mathrm{M}}>0$.

ELASTIC COLLISION:

As the system (1.1) is unnaturally restricted in solution domain, we need to consider the behaviour of solutions which approach $Y=0$ from above. We assume elastic behaviour at this boundary, with coefficient of restitution $0<C_{F}<1$. ; i.e., a trajectory 
having velocity $\mathrm{V}$ will reverse its direction, and assume new velocity $-\mathrm{C}_{\mathrm{F}} \mathrm{V}$. For the forced, fluid dynamic oscillator this corresponds to the ball striking the foreshield of the fan and rebounding with reduced velocity.

PHASE SPACE:

It becomes helpful to write equation (1.1) in the reduced form

$$
\begin{aligned}
& \dot{\mathrm{Y}}=\mathrm{V} \\
& \dot{\mathrm{V}}=\mathrm{F}(\mathrm{Y})-\mathrm{G}
\end{aligned}
$$

where $\left({ }^{*}\right)$ denotes the time derivative, and where $(\mathrm{V}, \mathrm{Y})$ are phase-plane variables. It is always assumed that $\mathrm{F}(\mathrm{Y})$ has adequate continuity properties to guarantee existence and uniqueness of solutions for any initial values considered and that the solutions may be continued for all time. We observe that $\left(0, \mathrm{Y}_{\mathrm{S}}\right)$ is the only singular point of the system (1.4) and recall that separate trajectories of the autonomous system (1.4) cannot cross each other. We shall denote by $\mathrm{Z}\left(\mathrm{V}_{0}, \mathrm{Y}_{0}\right)$ the trajectory which assumes the initial values indicated by the subscripts. From equation (1.3) and the monotonicity of $F(Y)$, we see that $V$ must increase with time if $Y_{0}<Y_{S}$, and decrease for $Y_{0}>Y_{S}$, as the trajectory is traversed.

MOTIVATION:

The purpose of this note is to study the periodic solutions of the above nonlinear system. We consider certain sets of attraction in the phase space and show that any trajectory leaving such a set must return to it in finite time. Trajectories which return to the same point are then, of necessity, periodic [1]. The symmetry properties of the system in conjunction with a non-contractive Picard iteration are used to show that an infinite family of such solutions exist. Indeed, trajectories having sufficient momentum to penetrate to the locus $\mathrm{Y}=0$ after sufficient dissipation of energy by boundary collisions will all approach arbitrarily near to a limiting periodic trajectory, $\Gamma_{L}$. We prefer to call such behavior eventually periodic motion. Our main result concerns the limit cycle, $\Gamma_{L}$, and may be stated as follows:

Theorem 1: The trajectory $\Gamma_{L}=Z(0,0)$ is a periodic limit cycle which separates the trajectories of system (1.4) into two classes:

I. All trajectories emanating from initial points exterior to $\Gamma_{L}$ are eventually periodic, approaching the limit cycle $\Gamma_{L}$ as $t$ increases without bound.

II. All trajectories emanating from points interior to $\Gamma_{L}$ close upon themselves in finite time and represent periodic motions.

\section{PRELIMINARY CONSIDERATIONS.}

We shall defer the proof of our central result, Theorem 1, until we have estabilished some useful supporting results:

Lemma 1. For $\mathrm{V}_{0}>0$, the mapping

$$
\mathrm{T}(\mathrm{y})=\mathrm{v}_{0}^{2} /(2 \mathrm{G})+\mathrm{Y}_{0}+\frac{1}{\mathrm{G}} \int_{\mathrm{Y}_{0}}^{\mathrm{Y}} \mathrm{F}(\mathrm{S}) \mathrm{ds}
$$

has at least one fixed point $\mathrm{Y}^{*}>\mathrm{Y}_{0}$.

Proof: Due to the monotonicity of $F(Y)$, the sequence

$$
\left\{\mathrm{Y}_{\mathrm{N}}: \mathrm{Y}_{\mathrm{N}+1}=\mathrm{T}\left(\mathrm{Y}_{\mathrm{N}}\right), \mathrm{N}=1,2,3, \ldots\right\},
$$

with $Y_{1}=Y_{0}$ (whence, $Y_{2}>Y_{1}$ ) is monotone increasing and bounded. Thus, the Picard 
iteration (2.2) must converge to a fixed point of (2.1). Monotonicity of the sequence guarantees that the fixed point is greater than the starting value. Q.E.D.

Comment 1. By integrating (1.5), we may interpret the fixed poing, $Y *$, as a crossing of the $\mathrm{Y}$-axis for a trajectory of equation (1.1) which emanates from $\mathrm{Z}\left(\mathrm{V}_{0}, \mathrm{Y}_{0}\right)$ with $\mathrm{V}_{0}$ positive.

Comment 2. It has been pointed out [2] that the monotonicity of $F(Y)$ and the positiveness of $G$, together with the peculiar functional form of system (1.4), are in themselves sufficient to guarantee, independently of Lemma 1 , that all trajectories of the set $\mathrm{S}=\left\{\Gamma: \Gamma=\mathrm{Z}_{1}\left(\mathrm{~V}_{0}, \mathrm{Y}_{0}\right), \mathrm{V}_{0}>0\right\}$ must eventually return to the $\mathrm{Y}$-axis.

Lemma 2: Any portion of a trajectory of (1.4) which penetrates both half-planes $Y>0$ and $\mathrm{Y}<0$, forms a connected set, and does not intersect the $\mathrm{V}$-axis.

Proof: This result follows from Lemma 1 of Kotin [3].

3. EXISTENCE OF PERIODIC SOLUTIONS.

The Soviet school of trajectory analysts have brought to bear on the study of phase plane trajectories the weapons of topological dynamics [4], featuring the ideas of wandering points, recurrent sets and central motions. Although we shall have no need for such generalities here, we borrow the following useful notions:

Definition $1: S$ is a set attraction for system(1.4) if any trajectory emanating initially from a point in $S$ either remains in $S$, or else returns to $S$ in finite time.

The finding of sets of attraction is a precusor to the determination of periodic solutions, as any trajectory whose initial point belongs to a set of attraction and which subsequently returns to the same point is, of necessity, periodic.

Theorem 2: The sets $S_{1}=\left\{(0, Y): 0<Y<Y_{S}\right\}$ and $S_{2}=\{(V, 0): V$ arbitrary $\}$ are sets of attraction for system (1.4).

Proof: (i) Set $\mathrm{S}_{1}$ is attractive: Consider the trajectory with initial values in $S_{1}$. Since $V$ is initially positive, it penetrates the top half-plane, to some point $Z\left(v_{0}, Y_{0}\right)$, with $\mathrm{V}_{0}$ nonvanishing. By Lemma 2, this trajectory must later intersect the $Y$-axis, at $\mathrm{Y}>\mathrm{Y}_{\mathrm{S}}$, where $\mathrm{V}$ is negative. As it must now penetrate the other half-plane, by Lemma 1 , it closes on itself, representing a periodic oscillation. (ii) Set $S_{2}$ is attractive: The proof parallels that of statement (i). However, now a trajectory $z\left(V_{0}, 0\right)$ does not close on itself, but after bouncing at $Y=0$ achieves the new starting values $\mathrm{Z}\left(\mathrm{C}_{\mathrm{F}} \times \mathrm{V}_{0}, 0\right)$. Hence, no trajectory of $\mathrm{S}_{2}$ is periodic.) Theorem 2 follows.

Proof of Theorem 1. I. Eventually Periodic Motions - As in the proof of Theorem 2, we recall that no trajectory of $\mathrm{S}_{2}$ is periodic. Since any point exterior to $\Gamma_{L}$ is eventually contained in the stream-tube formed by trajectories having initial point in $S_{2}$, no trajectory having initial point exterior to $\Gamma_{L}$ is periodic. Consider a stream-tube emanating from initial set $S_{R}=\left\{z\left(v_{0}, 0\right): 0<v_{0} \leqq R\right\}$. This stream-tube defines a oneto-one, continuous map

$$
H: Z\left(V_{0}, 0\right) \rightarrow Z\left(C_{F} \times V_{0}, 0\right)
$$

with the association obtained by following each trajectory through one bounce. After $N$ bounces, from

$$
H^{N}\left(S_{R}\right)=C_{F} \times S_{R}
$$

we see that

$$
\lim _{N \rightarrow \infty} H^{N}\left(S_{R}\right)=\{(0.0)\}
$$


i.e., every trajectory in $S_{2}$ approaches $\Gamma_{L}$ as a limit cycle. However, $R$ is arbitrary. II. Periodic Motions - By Theorem II, every trajectory emanating from $S_{1}$ is periodic. The stream-tube formed by such trajectories sweeps out $\Gamma_{L}$ and its interior, with the exception of the singular point, which is thus a center. Theorem I follows.

4. APPLICATIONS.

We now briefly consider a potential application of the previous abstract mathematical development. Returning to the ball suspended-in-fan problem, we shall obtain an approximation to the equation of motion. In the absence of a fan, the drag force on the ball can be determined from Reynolds principle of similarity. However, when the fan is blowing, two characteristic velocities for the flow exist, and it becomes difficult to model the drag forces in a simple way. However, we take the following approach: From cursory examination of the flow from a household hairdryer, we observe that the jet provides a fairly constant force in the immediate vicinity of the exit plane. Due to spreading of the jet this force rapidly diminishes, becoming negligible several diameters away. Schlichting [5] derives an inverse Y-dependency for the normal component of velocity of an infinitesmal circular jet. This leads to an inverse Y-square dependency for an effective instantaneous drag force resulting solely from the fan, of the form

$$
\mathrm{F}_{\text {Fan }}=\text { Constant* } \mathrm{K}(\mathrm{Y})^{2}
$$

where $\mathrm{Y}_{\mathrm{M}}>\mathrm{Y}_{\mathrm{N}}$ and

$$
K(y)= \begin{cases}\text { Constant, } Y<Y_{M} \\ 1 /\left(Y-Y_{N}\right), Y \geqq Y_{M}\end{cases}
$$

The basic forces on the ball are jet-induced form drag, gravity, buoyancy, and added mass [6] due to acceleration. Without solving a full three-dimensional fluid dynamics flow problem numerically, the exact drag interaction is uncertain. However, a qualitative approximation might be obtained by employing a force of the form [4.2], where we are now postulating such aneffective drag force from the fan as a first approximation to reality. We thus obtain an equation of motion of the form in equation (1.1).

For flow regimes in which no vortex shedding occurs on the back of the sphere, it would appear that an uncoupled fan drag force of the form required by equation (4.2) is plausible. The singular point of system (1.1) represents releasing the ball with zero velocity at a point in the flow where the forces from the jet balance the other forces represented by $G$.

of course, whenever additional damping of any sort is present in system $(1.1)$, no periodic solutions can exist; the motion eventually damps to the equilibrium position. However, in the case of near-inviscid fluids such as air, for vibrations of small amplitude the damping becomes almost negligible; over short periods of observation the motion then appears essentially periodic. Hence, system (1.1) affords a qualitative model of motion, assuming that the ball is spherically symmetric and homogenous in material makeup. Otherwise, spinning motions coupled with small processions can lead to angular momentum vector having nonvanishing component perpendicular to the primary direction of flow. Clearly, the affects of non-negligible Magnus forces leads to a problem which can no longer be considered essentially one-dimensional. 


\section{REFERENCES}

1. HEINBOCKEL, J. H., and STRUBLE, R. A., The Existence of Periodic Solutions of Nonlinear Oscillators, J. Soc. Indust. Appl. Math., 13, (1965) 6-37.

2. STRUBLE, R. A., Private Communication.

3. KOTIN, Leon, Solutions of Systems of Periodic Differential Equations, J. Math. Anal. and Appli., $\underline{8}$, (1964) 52-56.

4. NEMYTSKII, V. V., and STEPANOV, V. V., Qualitative Theory of Differential Equations.

5. SCHLICHTING, Herman, Boundary Layer Theory, McGraw-Hil1, New York, 1977.

6. LAMB, Sir Horace, Hydrodynamics, Dover, 1945. 


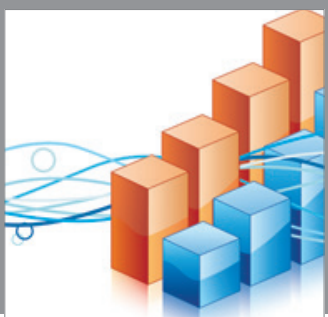

Advances in

Operations Research

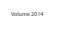

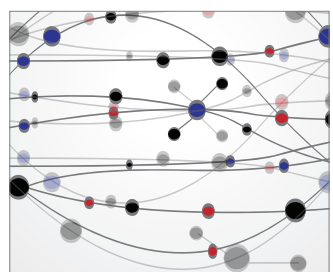

\section{The Scientific} World Journal
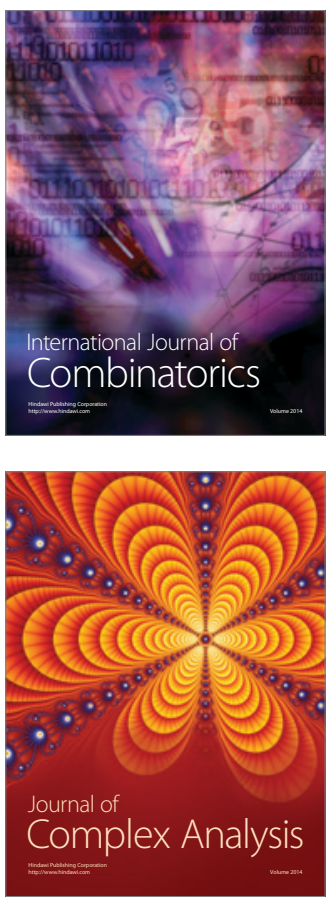

International Journal of

Mathematics and

Mathematical

Sciences
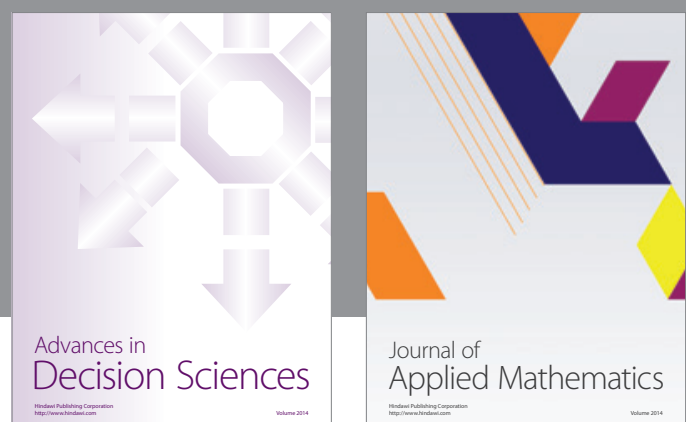

Journal of

Applied Mathematics
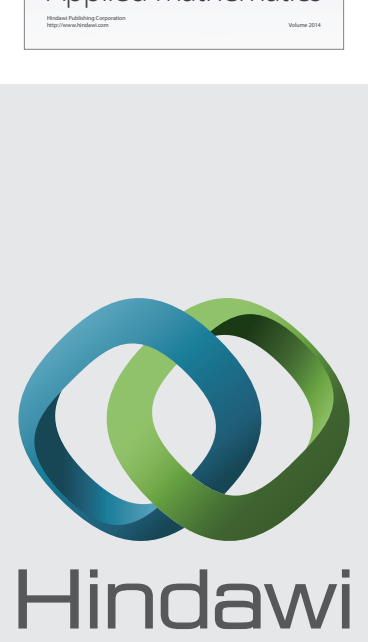

Submit your manuscripts at http://www.hindawi.com
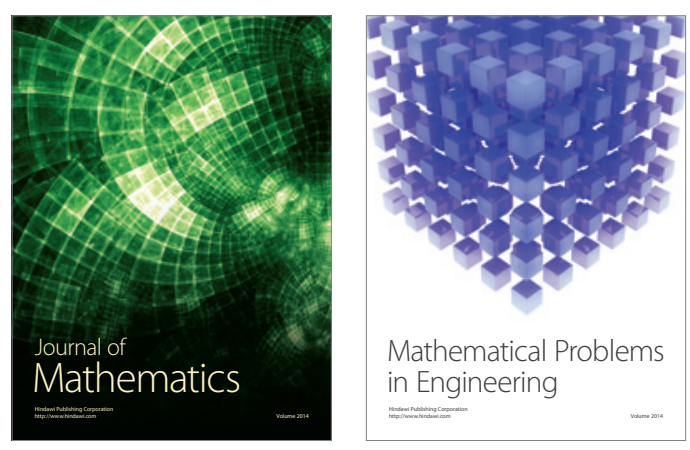

Mathematical Problems in Engineering
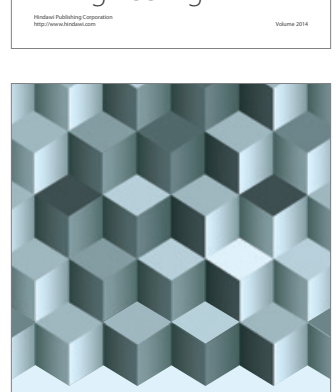

Journal of

Function Spaces
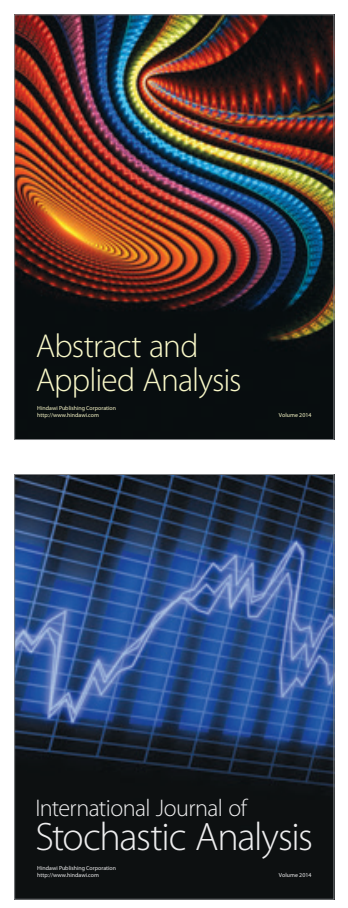

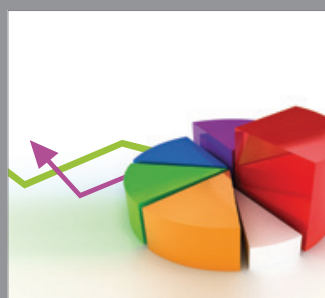

ournal of

Probability and Statistics

Promensencen
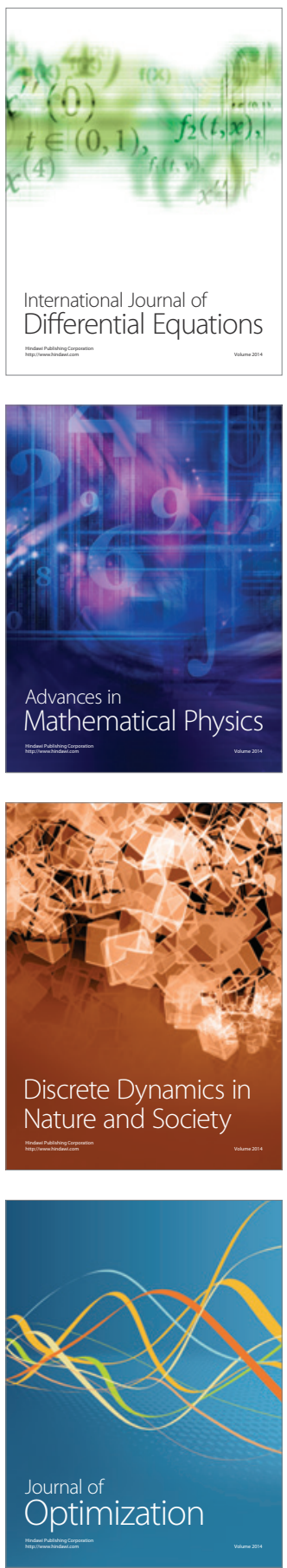